\title{
Synthetic Mechanochemical Molecular Swimmer
}

\author{
Ramin Golestanian* \\ Department of Physics and Astronomy, University of Sheffield, Sheffield S3 $7 R H, U K$ and \\ Laboratoire de Physico-Chimie Théorique, UMR CNRS Gulliver 7083, ESPCI, 75231 Paris Cedex 05, France
}

(Dated: October 31, 2018)

\begin{abstract}
A minimal design for a molecular swimmer is proposed that is a based on a mechanochemical propulsion mechanism. Conformational changes are induced by electrostatic actuation when specific parts of the molecule temporarily acquire net charges through catalyzed chemical reactions involving ionic components. The mechanochemical cycle is designed such that the resulting conformational changes would be sufficient for achieving low Reynolds number propulsion. The system is analyzed within the recently developed framework of stochastic swimmers to take account of the noisy environment at the molecular scale. The swimming velocity of the device is found to depend on the concentration of the fuel molecule according to the Michaelis-Menten rule in enzymatic reactions.

PACS numbers: 87.19.rs, 07.10.Cm, 82.39.-k
\end{abstract}

One of the aims of nanotechnology is to be able to make synthetic molecular devices that could propel themselves through fluidic environments and perform targeted tasks such as delivery of therapeutic agents or carrying out mechanical work [1]. At such small scales, one cannot apply standard deterministic strategies used in engineering at the macroscopic scales, as the dynamics of any device will be overwhelmed by fluctuations of thermal or other origins. The right strategy is to find a way to impose a bias on these fluctuations such that they will average out to our desired behavior, as can be learned from the example of biological molecular motors [2].

In addition to enduring the noisy environment, molecular swimmers would also need to overcome the fundamental difficulty posed by the governing hydrodynamic rules at low Reynolds number conditions: they should utilize a non-reciprocal sequence of deformations to break the time reversal symmetry in their periodic motions and achieve swimming [3]. This is a nontrivial task if one is to use a minimal set of degrees of freedom [4 11], which will most likely be what can be afforded in a humanengineered device.

There has been a significant recent development along this line with the advent of a number of experimentally made micron-scale prototypes of such low Reynolds number swimmers. Using magnetic actuation of micron-sized linked magnetic beads 12, 13 or manipulation of particles using optical tweezers [14], it was demonstrated that low Reynolds number propulsion can be achieved at the micro-scale via non-reciprocal motion. In these experiments, the actuation mechanisms were externally enforced (using oscillating magnetic fields or optical traps), which means that strictly speaking the devices were not self-propelled swimmers. Moreover, it is not clear if such actuation mechanisms could be scaled down to be applicable to molecular devices. This means that we are in need of new strategies to be able to program nonreciprocal molecular deformations.

While other external triggers - such as laser pulses that could induce conformational changes - could in principle be pursued [1], it would be ideal to be able to couple the conformational changes to a local source of free energy via a chemical reaction, like motor proteins [15]. This will allow the mechanism to potentially accommodate arbitrary degrees of complexity, and avoid interference between different parts of the system. To my knowledge, this scenario has not been considered so far in the literature for microscopic swimmers, although there have been demonstrations of mechanical actuation of elastic gels by using oscillatory chemical reactions (at macroscopic scales) [16]. A strategy based on catalysis of a chemical reaction has already been used for making self-propelled colloidal particles, which utilize non-equilibrium interfacial (phoretic) interactions towards their net motion [17, 18]. While this mechanism of motility has shown to be extremely powerful, exploring other possible strategies would help create a diverse range of approaches that could be used in the future to optimize devices for specific purposes and overcome strategy-specific hurdles for a particular end use. For example, a swimmer that utilizes interfacial interactions as its main motility mechanism is potentially vulnerable to intervention by other interfacial forces when it is close to boundaries or other devices. Moreover, one wonders why nature has not chosen to take advantage of the "phoretic" strategy in any living system; one may speculate that it could be because it cannot be conveniently miniaturized and robustly function in maximally crowded intracellular environments.

Here I propose an alternative minimal design for a molecular swimmer, which would be able to catalyze chemical reactions and use the free energy gain and products to induce the conformational changes that would be sufficient for achieving low Reynolds number propulsion in a noisy environment [19]. The present study could also shed some light on how the motor proteins could combine enzymatic action with conformational changes, as it presents a full physical analysis of a simple model that explains how such a coupling could work in practice. 


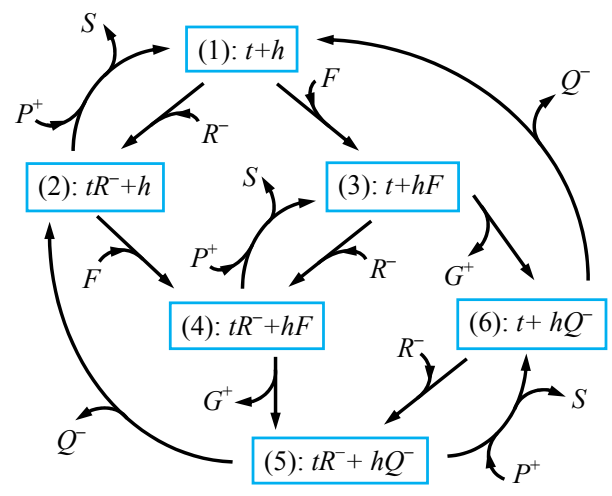

FIG. 1. The 6-state chemical cycle of the three-sphere molecular device that comprises enzyme $h$ at the head and enzyme $t$ at the tail (see Fig. 2). $h$ catalyzes $F \rightarrow Q^{-}+G^{+}$and $t$ catalyzes $R^{-}+P^{+} \rightarrow S$.

The proposed design for the molecular swimmer is based on the simple three-sphere low Reynolds number swimmer model [5, 20]. To incorporate catalytic activity, we take two enzymes, $h$ and $t$, and place them at the head and the tail of the three-sphere device, respectively. The enzymes catalyze the following chemical reactions

$$
\begin{aligned}
& F+h \rightarrow h F \rightarrow h Q^{-}+G^{+} \rightarrow h+Q^{-}+G^{+}, \\
& R^{-}+P^{+}+t \rightarrow t R^{-}+P^{+} \rightarrow t+S,
\end{aligned}
$$

which would otherwise occur very slowly in the bulk. Since the head can be found in three distinct states of $h, h F$, and $h Q^{-}$, and the tail has two distinct states of $t$ and $t R^{-}$, the combined system can have 6 distinct chemical states. The reactions (1) and (2) then multiply within these states and produce the chemical cycle shown in Fig. 1]

The second ingredient in the design is to take advantage of the presence of ionic components in the reactions and use electrostatic interactions to induce conformational changes in the swimmer. If we make the middle sphere permanently negatively charged (see Fig. 2), the temporarily charged states of $h Q^{-}$and $t R^{-}$will introduce electrostatic repulsion that can elongate the right and left arms, respectively. This allows us to map the 6 chemical states shown in Fig. 1 onto the 6 distinct mechanochemical states shown in Fig. 2. These mechanochemical states can be represented in a 3D configuration space whose axes correspond to deformation of the right $\operatorname{arm} U_{R}$, deformation of the left $\operatorname{arm} U_{L}$, and complexation of $h$ with $F$. The reaction routes in the chemical cycle in Fig. 11 can now be used to identify the corresponding transitions between the mechanochemical states in the configuration space, as shown in Fig. 2. The relevant transition rates are defined on the configuration space diagram in Fig. 2. I have chosen a notation where the rates of similar transitions are differentiated by a prime, with the general rule that $k_{\alpha}^{\prime}>k_{\alpha}$. These
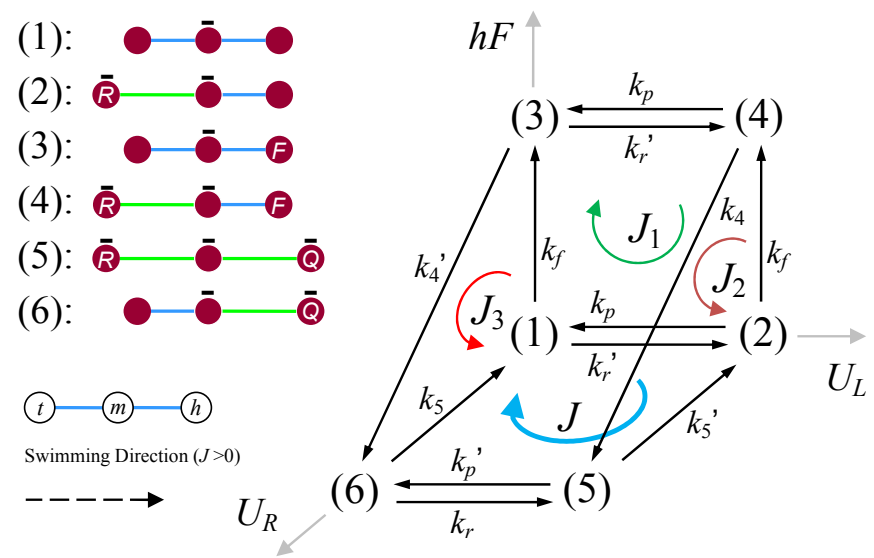

FIG. 2. The 6 distinct mechanochemical states of the threesphere swimmer in the configuration space. The conformational states of the swimmer are determined by the projection onto the space of the deformation of the left $\left(U_{L}\right)$ and right $\left(U_{R}\right)$ arms, while binding of $h$ with $F$ produces distinct chemical states that have identical conformations. All possible transitions described in the chemical cycle in Fig. 1 are shown by arrows with attributed rate constants.

choices are made depending on whether the presence of additional electrostatic charges hampers or facilitates a certain reaction 21].

We do not make assumptions about any specific ordering of the various events involved in the cycle (see Figs. 1 and 2). For example, state (1) can proceed to state (2) upon binding of $R^{-}$to $t$, and can also proceed to (3) if $F$ binds to $h$. Any of these two can happen at a time in a stochastic manner, and only the average rates of these transitions are controlled. Moreover, not all of the transitions contribute constructively towards net swimming. For example, state (2) can proceed to state (4) upon binding of $F$ to $h$, which will take the system further down towards completing a non-reciprocal mechanical cycle, but could also go back to state (1) if $P^{+}$binds to $t R^{-}$, which undoes the progress made by the previous transition towards net swimming.

The dynamics of the device can be studied using a stochastic description of the conformational transitions and the relative population of the different states at stationary state [20]. In stationary situation, each of the six mechanochemical states shown in Fig. 2 is populated with a probability $p_{i}(i=1, \cdots, 6)$, subject to the normalization condition $p_{1}+p_{2}+p_{3}+p_{4}+p_{5}+p_{6}=1$. The loops in the configuration space contribute probability currents denoted as $J, J_{1}, J_{2}$, and $J_{3}$ in Fig. 2. Conservation of probability current leads to the following nine equations between the probabilities and the currents: $J+J_{3}=k_{5} p_{6}, J_{3}+J_{1}=k_{f}[F] p_{1}, J_{3}=$ $k_{4}^{\prime} p_{3}, J_{2}-J=k_{5}^{\prime} p_{5}, J_{2}-J_{1}=k_{f}[F] p_{2}, J_{2}=k_{4} p_{4}$, $J_{1}=k_{r}^{\prime}\left[R^{-}\right] p_{3}-k_{p}\left[P^{+}\right] p_{4}, J=k_{p}^{\prime}\left[P^{+}\right] p_{5}-k_{r}\left[R^{-}\right] p_{6}$, $J-J_{1}=k_{r}^{\prime}\left[R^{-}\right] p_{1}-k_{p}\left[P^{+}\right] p_{2}$. The above ten linear 


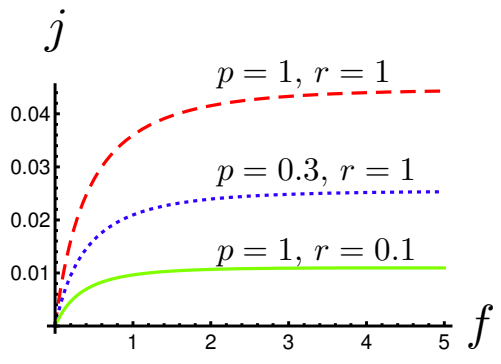

(a)

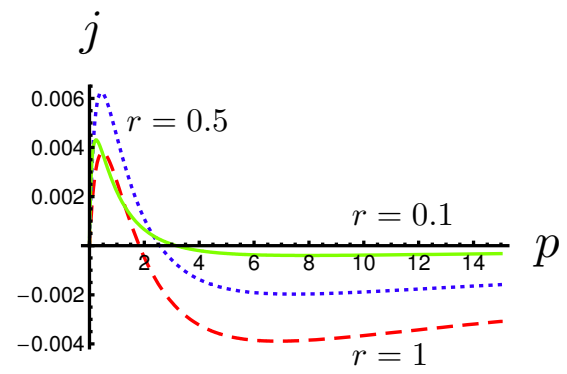

(b)

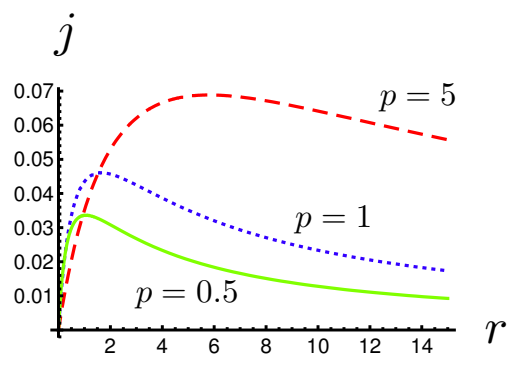

(c)

FIG. 3. Dimensionless current $j=J / k_{5}$ as a function of $f=k_{f}[F] / k_{5}, r=k_{r}\left[R^{-}\right] / k_{5}$, and $p=k_{p}\left[P^{+}\right] / k_{5}$, corresponding to $\alpha=1.5, \beta=1.5, \delta=1.1$, and $b=1$. (a) Michaelis-Menten behavior, for $\gamma=1.1$. (b) Sign change, for $f=1$ and $\gamma=2.7$. (c) Nonmonotonic concentration dependence, for $\gamma=1.1$ and $f \rightarrow \infty$.

equations can be solved for the ten unknowns (six probabilities and four currents) as functions of the concentrations $[F],\left[R^{-}\right]$, and $\left[P^{+}\right]$.

The six states in the mechanochemical configuration space of the swimmer correspond to only four independent conformations (Fig. 2). To study the coupling between the kinetics of the mechanochemical cycle and the hydrodynamics of the ambient fluid, we need to consider only the transitions between the distinct conformational states. Assuming that the extensions of the arms are small compared to their equilibrium lengths, it has been shown that the net swimming velocity of the device is controlled by the surface area $A$ enclosed by the conformational states that make the $(1) \rightleftharpoons(2) \leftarrow(5) \rightleftharpoons(6) \rightarrow$ (1) loop (in Fig. 2) and the net rate of sweeping this area $J$ [20]. If we choose to make all the rates dimensionless by $k_{5}$, and in particular define the dimensionless current $j=J / k_{5}$, we can write the average swimming velocity of the device as

$$
V=\frac{g D A k_{5}}{L^{2}} j\left(\frac{k_{f}[F]}{k_{5}}, \frac{k_{r}\left[R^{-}\right]}{k_{5}}, \frac{k_{p}\left[P^{+}\right]}{k_{5}}\right),
$$

where $g$ is a geometrical numerical prefactor of order unity [22], $D$ is the diameter of the spheres, and $L$ is the average total length of the swimmer.

For simplicity, let us define the dimensionless variables $\alpha=k_{r}^{\prime} / k_{r}, \beta=k_{p}^{\prime} / k_{p}, \gamma=k_{4}^{\prime} / k_{4}, \delta=k_{5}^{\prime} / k_{5}$, and $b=k_{4} / k_{5}$, as well as the dimensionless concentrations $f=k_{f}[F] / k_{5}, r=k_{r}\left[R^{-}\right] / k_{5}$, and $p=k_{p}\left[P^{+}\right] / k_{5}$. As argued above, we expect $\alpha, \beta, \gamma$, and $\delta$ to be numerical factors (not too much) larger than unity. Solving the above system of equations, we find that detailed-balance holds (i.e. $j=0$ ), when

$$
\gamma(\alpha \beta-\delta)+(\alpha \beta-\gamma \delta)(f+p+\alpha r)=0 .
$$

By tuning the concentrations $f, r$, and $p$ away from values that satisfy the above equality, we can drive the system away from equilibrium and obtain a nonvanishing swimming velocity $(j \neq 0)$.
The concentration dependence of $j$ reveals a number of interesting features in the device, as shown in Fig. 3. Figure 3 a shows that the dependence of $j$ and consequently the swimming velocity on the concentration of $F$ adopts a Michaelis-Menten form. This behavior, which is also observed in molecular motors [15], is inherited from the (multi-step enzymatic) reaction kinetics. Figure $3 b$ shows that for sufficiently low concentrations of $F$ and relatively large values of $\gamma$, it is possible to have a situation where $j$ changes sign, which leads to the reversal of the swimming velocity. This can be understood from the condition of detailed-balance in Eq. (4) as $j$ is proportional to its left hand side. Figure 3 c shows that when the concentration of $F$ is at its saturation limit (see Fig. 3a) the dependence of $j$ on the concentrations of $R^{-}$and $P^{+}$is nonmonotonic, and suggests a strategy to optimize the swimming velocity.

There are many efficient enzymatic reactions that could be used in practice in such a setup. For example, Eq. (11) can represent the conversion of carbon dioxide $\left(F=\mathrm{CO}_{2}\right)$ (plus water) into bicarbonate $\left(Q^{-}=\mathrm{HCO}_{3}^{-}\right)$ and proton $\left(G^{+}=\mathrm{H}^{+}\right)$catalyzed by carbonic anhydrase $(h=\mathrm{CA})$ [23]. An example for Eq. (2) could be superoxide anion $\left(R^{-}=\mathrm{O}_{2} \cdot^{-}\right)$being scavenged by $s u$ peroxide dismutase $(t=\mathrm{SOD})$ by consuming a proton $\left(P^{+}=\mathrm{H}^{+}\right)$and producing hydrogen peroxide and oxygen $\left(S=\frac{1}{2} \mathrm{H}_{2} \mathrm{O}_{2}+\frac{1}{2} \mathrm{O}_{2}\right)$ [23]. Looking at Figs. 1$]$ and 2. we find that in this example $k_{5}=k_{\mathrm{CA}}=6 \times 10^{5} \mathrm{~s}^{-1}$ [23], while $k_{r}=k_{\text {SOD }} \sim 3 \times 10^{9} \mathrm{M}^{-1} \mathrm{~s}^{-1}$ [24]. While we need to know all of the rates to be able to perform a full calculation, it is possible to make a rough estimate by looking at typical optimal values of $j \sim 10^{-2}$ from Fig. 3, and the above estimate for $k_{5}$. Using Eq. (3) with $D \sim 1 \mathrm{~nm}, A \sim 1 \mathrm{~nm}^{2}$, and $L \sim 10 \mathrm{~nm}$, we find an estimate of $V \sim 100 \mathrm{~nm} \mathrm{~s}^{-1}$, which is comparable to the efficient molecular motors that use protofilament tracks for their locomotion. In this molecular realization, these enzymes could presumably play the role of the spheres themselves, and could be attached to one another using 
unstructured tails or covalently bonded polymeric tethers. It might also be feasible to attempt a larger scale setup with colloidal spheres covered with adsorbed enzymes and connected with polymeric tethers.

In practice it might be difficult to fully suppress other transitions, and we might have to deal with more complicated configurational spaces (e.g. involving other transition routes between these states) as well as the back reactions. Moreover, realistic chemical reactions might have more complicated kinetics (such as the four-stage kinetics of the reaction of superoxide anion by SOD [23] that we have simplified into two stages in the above example). Such complications can be taken into account in the calculation of the average swimming velocity using a similar strategy [25], although they are not expected to change the qualitative behavior of the system provided the detailed-balance is broken.

In addition to affecting the conformational transitions, thermal fluctuations will also tend to randomize the orientation of the swimmer, on time scales larger than the rotational diffusion time $\tau_{r} \sim \eta L^{3} /\left(k_{\mathrm{B}} T\right)$, where $\eta$ is the viscosity of water, $k_{\mathrm{B}}$ is the Boltzmann constant and $T$ is the temperature. In addition to the rotational diffusion of the swimmer, fluctuations in the density of the fuel molecule could also induce anomalous fluctuations in the magnitude and direction of the velocity. The combination of these effects could lead to a variety of different behaviors, similar to those found in the context of selfphoretic swimmers [26]. We note that a related enhanced diffusion of active enzymes has recently been observed experimentally [27]. The dependence of the velocity of the swimmer on concentration of the fuel in Eq. (3) also suggests that such artificial swimmers could be guided using chemotaxis [18, 28, 29].

In conclusion, I have proposed a design for a molecular scale low Reynolds number swimmer that can use the free energy gain and products of a nonequilibrium chemical reaction and undergo conformational changes that are non-reciprocal and therefore can lead to net swimming under low Reynolds number conditions. While the present analysis has used a minimal kinetic model that can achieve swimming at such small scales, the scheme can be readily generalized to help analyze any realistic mechanochemical cycle corresponding to more complex molecular devices that could be synthesized.

I would like to thank A. Ajdari, D. Lacoste, A. Najafi, E. Raphaël, and H. A. Stone for fruitful discussions. This work was supported by CNRS and EPSRC.

\footnotetext{
* r.golestanian@sheffield.ac.uk
}

[1] E. R. Kay, D. A. Leigh, and F. Zerbetto, Angew. Chem. Int. Ed. 46, 72 (2007); W. R. Browne and B. L. Feringa, Nature Nanotech. 1, 25 (2006).
[2] F. Julicher, A. Ajdari, and J. Prost, Rev. Mod. Phys. 69, 1269 (1997); R. D. Astumian, Science 276, 917 (1997); M. E. Fisher and A. B. Kolomeisky, Proc. Natl. Acad. Sci. (USA) 98, 7748 (2001).

[3] E. M. Purcell, Am. J. Phys. 45, 3 (1977); A. Shapere and F. Wilczek, Phys. Rev. Lett. 58, 2051 (1987); E. Lauga and T. Powers, Rep. Prog. Phys. 72, 096601 (2009).

[4] L. E. Becker, S. A. Kochler, and H. A. Stone, J. Fluid Mech. 490, 15 (2003).

[5] A. Najafi and R. Golestanian, Phys. Rev. E 69, 062901 (2004).

[6] J. E. Avron, O. Gat, and O. Kenneth, Phys. Rev. Lett. 93, 186001 (2004).

[7] R. Dreyfus, J. Baudry, and H. A. Stone, Eur. Phys. J. B 47, 161 (2005).

[8] B. U. Felderhof, Phys. Fluids 18, 063101 (2006).

[9] D. Tam and A. E. Hosoi, Phys. Rev. Lett. 98, 068105 (2007).

[10] C. M. Pooley, G. P. Alexander, and J. M. Yeomans, Phys. Rev. Lett. 99, 228103 (2007).

[11] S. Günther and K. Kruse, Europhys. Lett. 84, 68002 (2008).

[12] R. Dreyfus et al., Nature 437, 862 (2005).

[13] P. Tierno et al., Phys. Rev. Lett. 101, 218304 (2008).

[14] M. Leoni et al., Soft Matter 5, 472 (2009).

[15] J. Howard, Mechanics of Motor Proteins and the Cytoskeleton (Sinauer, New York, 2000).

[16] J. R. Howse et al., Nano Lett. 6, 73 (2006).

[17] P. Lammert, J. Prost, and R. Bruinsma, J. Theor. Biol. 178, 387 (1996); W. F. Paxton et al., J. Am. Chem. Soc. 126, 13424 (2004); W. F. Paxton et al., Angew. Chem. Int. Ed. 45, 5420 (2006); S. Fournier-Bidoz et al., Chem. Comm. 441 (2005); N. Mano and A. Heller, J. Am. Chem. Soc. 127, 11574 (2005); R. Golestanian, T. B. Liverpool, and A. Ajdari, Phys. Rev. Lett. 94, 220801 (2005); G. Rückner and R. Kapral, Phys. Rev. Lett. 98, 150603 (2007).

[18] J. R. Howse et al., Phys. Rev. Lett. 99, 048102 (2007).

[19] The studies of Refs. [17, 18] rely on coupling of chemical reactions with the mechanical motion of fluid relative to the colloid, and are in contrast with what I term as "mechanochemical" here, which refers to change in the morphology of the swimmer due to chemical reactions.

[20] R. Golestanian and A. Ajdari, Phys. Rev. Lett. 100, 038101 (2008).

[21] For example, the transitions $(6) \rightarrow^{k_{5}}$ (1) and (5) $\rightarrow^{k_{5}^{\prime}}(2)$ both involve releasing a $Q^{-}$from $h$, but $k_{5}^{\prime}$ corresponds to the case where the tail is in $t R^{-}$form, which should facilitate the release due to electrostatic repulsion as compared to when the tail is unoccupied; thus we expect that $k_{5}^{\prime}>k_{5}$.

[22] R. Golestanian and A. Ajdari, Phys. Rev. E 77, 036308 (2008).

[23] J. M. Berg, J. L. Tymoczko, and L. Stryer, Biochemistry (W.H. Freeman and Co., New York, 2002).

[24] C. Bull, and J. A. Fee, J. Am. Chem. Soc. 107, 3295 (1985); J. Biol. Chem. 261, 13000 (1986).

[25] R. Golestanian and A. Ajdari, J. Phys.: Condens. Matter 21, 204104 (2009).

[26] R. Golestanian, Phys. Rev. Lett. 102, 188305 (2009).

[27] H. S. Muddana et al., J. Am. Chem. Soc. 132, 2110 (2010).

[28] Y. Hong et al., Phys. Rev. Lett. 99, 178103 (2007).

[29] B. Abecassis et al., Nature Mat. 7, 785789 (2008). 